\title{
Prediction, Suppression of Visual Response, and Modulation of Visual Perception: Insights From Visual Evoked Potentials and Representational Momentum
}

OPEN ACCESS

Edited by:

István Winkler,

Hungarian Academy of Sciences

(MTA), Hungary

Reviewed by:

István Czigler,

Hungarian Academy of Sciences

(MTA), Hungary

Kairi Kreegipuu,

University of Tartu, Estonia

*Correspondence:

Motohiro Kimura

m.kimura@aist.go.jp

Specialty section:

This article was submitted to

Cognitive Neuroscience,

a section of the journal

Frontiers in Human Neuroscience

Received: 25 June 2021

Accepted: 27 July 2021

Published: 25 August 2021

Citation

Kimura M (2021) Prediction, Suppression of Visual Response, and Modulation of Visual Perception:

Insights From Visual Evoked

Potentials and Representational

Momentum.

Front. Hum. Neurosci. 15:730962.

doi: 10.3389/fnhum.2021.730962

\section{Motohiro Kimura*}

Department of Information Technology and Human Factors, National Institute of Advanced Industrial Science and Technology (AIST), Tsukuba, Japan

When a visual object changes its position along with certain sequential regularities, the visual system rapidly and automatically forms a prediction regarding the future position of the object based on the regularities. Such prediction can drastically alter visual perception. A phenomenon called representational momentum (RM: a predictive displacement of the perceived final position of a visual object along its recent regular pattern) has provided extensive evidence for the predictive modulation of visual perception. The purpose of the present study was to identify neural effects that could explain individual differences in the strength of the predictive modulation of visual perception as measured by RM. For this purpose, in two experiments with a conventional RM paradigm where a bar was discretely presented in a regular rotation manner (with a step of $18^{\circ}$ in Experiment 1 and a step of $20^{\circ}$ in Experiment 2), visual evoked potentials (VEPs) in response to the regularly rotated bar were measured, and correlations between the magnitudes of RM and VEPs were examined. The results showed that the magnitudes of RM and central P2 were negatively correlated, consistently in both experiments; participants who showed a smaller central P2 tended to exhibit greater RM. Together with a previous proposal that central P2 would represent delayed reactivation of lower visual areas around the striate and prestriate cortices via reentrant feedback projections from higher areas, the present results suggest that greater suppression of delayed reactivation of lower visual areas (as indicated by smaller central P2) may underlie stronger predictive modulation of visual perception (as indicated by greater RM).

Keywords: visual evoked potentials (VEPs), representational momentum, visual perception, prediction suppression, central P2, individual difference

\section{INTRODUCTION}

Visual objects in the environment (e.g., a flying ball) dynamically change their positions. However, when an object's image hits an observer's eyes, the observer cannot perceive the image instantaneously; it takes about a tenth of a second after the image hits the eyes. Therefore, by the time the observer has perceived the object at a certain position, its actual position has already 
changed. Despite this fundamental problem, an observer can effortlessly interact with such objects in real time (e.g., by catching a flying ball). A possible solution to the problem of how the visual system can bridge the gap between perception and action is to form a prediction about the future position of the object, based on sequential regularities in the recent past (i.e., recent trajectory of the ball) (Mackay, 1958; Freyd, 1992; Nijhawan, 1994; Hubbard, 1995, 2005).

A phenomenon known as representational momentum (RM: Freyd and Finke, 1984, 1985) provides strong evidence for the existence of such prediction based on sequential regularities in the recent past and demonstrates that visual perception can indeed be strongly modulated by the prediction. RM denotes predictive displacement of the perceived final position of a changing object. In a conventional RM paradigm developed by Freyd and Finke (1984, 1985), participants observe a stimulus sequence where a bar is discretely presented in a regular rotation manner (denoted "inducing stimuli": e.g., $10^{\circ} / 30^{\circ} / 50^{\circ}$ ). Participants are required to compare the orientation of the final inducing stimulus (i.e., $50^{\circ}$ ) to that of a subsequent bar (denoted "probe"). It has been shown that participants report "same" with higher probability when the probe is slightly shifted forward along the regular direction of rotation (e.g., $52^{\circ}$ ) than when it is truly the same $\left(50^{\circ}\right)$ or shifted backward (e.g., $48^{\circ}$ ) (Freyd and Finke, 1985). RM is thought to reflect predictive displacement of the sensory representation of an object along its recent change pattern (Freyd, 1992; Hubbard, 1995, 2005). RM can be observed based on sequential regularities in position or orientation but also in other visual features (Kelly and Freyd, 1987; Hayes and Freyd, 2002) and sequential regularities in auditory features (Freyd et al., 1990), suggesting that the predictive displacement of sensory representation would be a general phenomenon across visual features and sensory modalities. Also, RM can occur without the observer paying much attention to the object (Hayes and Freyd, 2002; for related findings, see Finke and Freyd, 1985), suggesting that the predictive displacement of sensory representation can occur in an automatic and obligatory manner.

Representational momentum is a robust phenomenon that is stably observed across participants (Freyd and Finke, 1985). However, there seem to be large individual differences in the magnitude of RM (Finke et al., 1986; Verfaillie and d'Ydewalle, 1991), which leads to the assumption that there may be large individual differences in the strength of the predictive modulation of visual perception. The purpose of the present study was to identify neural effects that could explain individual differences in the strength of predictive modulation of visual perception as measured by RM. For this purpose, the present study measured visual evoked potentials (VEPs) with a conventional RM paradigm (Freyd and Finke, 1984, 1985). In two experiments, a bar was discretely presented in a regular rotation manner (i.e., inducing stimuli); with a step of $18^{\circ}$ in Experiment 1 (Figure 1) and $20^{\circ}$ in Experiment 2 (Figure 2). Participants were required to compare the orientation of the final (i.e., tenth) inducing stimulus to that of a subsequent probe. VEPs in response to inducing stimuli were measured, and correlations were examined between the magnitudes of RM and VEPs: (1) occipito-temporal P1 at around $110 \mathrm{~ms}$, (2) frontal N1 at around
$140 \mathrm{~ms}$, (3) occipito-temporal N1 at around $170 \mathrm{~ms}$, and (4) central P2 at around $200 \mathrm{~ms}$ after stimulus onset (Clark et al., 1995; Di Russo et al., 2002; Capilla et al., 2016). ${ }^{1}$

No previous study has examined the relationship between the magnitudes of RM and VEPs in response to inducing stimuli. However, based on a previous VEP finding on automatic prediction based on sequential regularities (Kimura and Takeda, 2015), the neural effect that is most likely to correlate with RM is central P2. To identify neural effects that specifically emerge when the current position of an object successfully matches the predicted position of the object based on sequential regularities, Kimura and Takeda (2015) compared VEPs elicited by bars that were discretely presented in a regular rotation manner (e.g., $10^{\circ} / 30^{\circ} / 50^{\circ} / 70^{\circ} / 90^{\circ} \ldots$, where the upcoming orientation of the bar could be predicted, as in the RM paradigm) to VEPs elicited by the same bars that were discretely presented in a random manner (e.g., $70^{\circ} / 10^{\circ} / 30^{\circ} / 90^{\circ} / 50^{\circ} \ldots$, where a prediction of the upcoming orientation of the bar could not be formed). It was found that central P2 at around $200 \mathrm{~ms}$ after stimulus onset was selectively suppressed when the upcoming orientation could be predicted compared to when a prediction could not be formed. Contrary to central P2, no difference in this comparison was found for occipito-temporal P1 at around $110 \mathrm{~ms}$, frontal N1 at around $140 \mathrm{~ms}$, and occipito-temporal N1 at around $170 \mathrm{~ms}$; instead, occipito-temporal P1 and N1 (but not frontal N1) were found to be suppressed only when bars were presented in a repetitive manner (e.g., $10^{\circ} / 10^{\circ} / 10^{\circ} / 10^{\circ} / 10^{\circ} \ldots$. ) compared to when bars were presented in a random manner (e.g., $70^{\circ} / 10^{\circ} / 30^{\circ} / 90^{\circ} / 50^{\circ} \ldots$ ), suggesting that these P1 and N1 effects represent repetition suppression attributable to stimulusspecific adaptation or neural refractoriness rather than prediction suppression (cf. Todorovic and de Lange, 2012). Therefore, at least when a regularly rotating bar was used as stimuli, the suppression of central P2 is thought to be a unique neural effect that could emerge when the current position of a visual object successfully matches the predicted position of the object based on sequential regularities.

The neural sources of central P2 at around $200 \mathrm{~ms}$ were previously localized in lower visual areas around the striate and prestriate cortices (Capilla et al., 2016); although the neural

\footnotetext{
${ }^{1}$ In general, VEPs time-locked to visual stimulus onset are comprised of (1) occipital $\mathrm{C} 1$ that peaks at around $60 \mathrm{~ms}$, (2) occipito-temporal P1 that peaks at around $110 \mathrm{~ms}$, (3) frontal $\mathrm{N} 1$ that peaks at around $140 \mathrm{~ms}$, (4) occipito-temporal $\mathrm{N} 1$ that peaks at around $170 \mathrm{~ms}$, and (5) central P2 that peaks at around $200 \mathrm{~ms}$ after stimulus onset (Clark et al., 1995; Di Russo et al., 2002; Capilla et al., 2016). The main neural sources of these VEPs were localized in the visual areas and the related areas belonging to the dorsal and ventral processing streams: (1) C1 in the striate cortex (i.e., V1), (2) P1 in the dorsal and ventral extrastriate cortices (e.g., V3 and V4), (3) frontal N1 in the parieto-occipital cortex, (4) occipito-temporal N1 in the dorsal extrastriate cortex (e.g., V3), and (5) P2 in the striate and prestriate cortices (i.e., V1 and V2) (Clark et al., 1995; Di Russo et al., 2002, 2003, 2008; Capilla et al., 2016). Although tentative, occipital C1, occipito-temporal P1, and frontal N1 may mainly represent early bottom-up activation, whereas occipitotemporal N1 and central P2 may mainly represent delayed reactivation of visual areas via reentrant feedback projections from higher areas (Di Russo et al., 2003, 2008; Capilla et al., 2016; for related findings, see Olson et al., 2001; Noesselt et al., 2002). Note that occipital C1 was not analyzed in the present study, since centrally presented visual stimuli (see Figures 1,2 ) were not suitable to observe $\mathrm{C} 1$; retinotopically specific single-quadrant stimulation is required to observe $\mathrm{C} 1$ (Clark et al., 1995).
} 


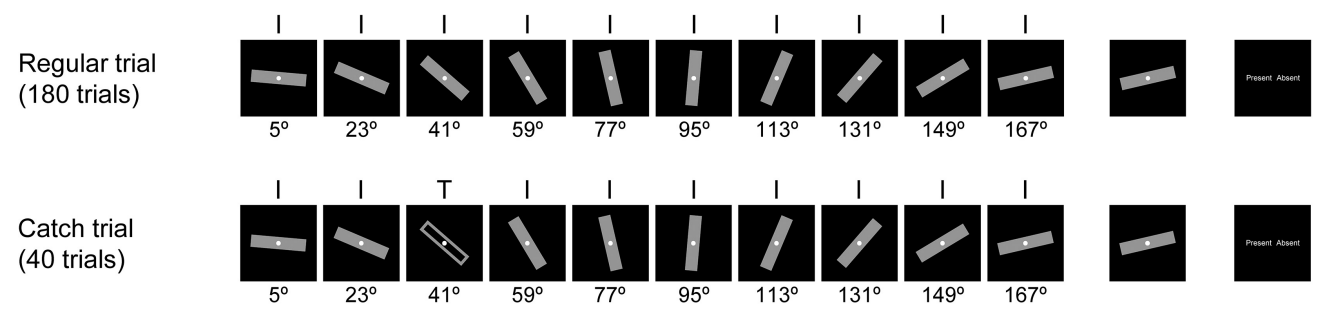

I: Inducing stimulus T: Target stimulus

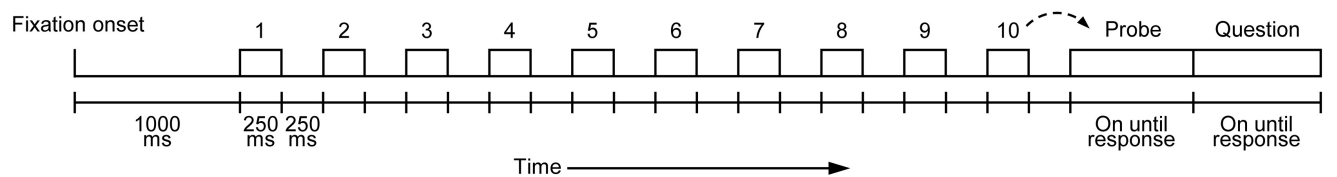

FIGURE 1 | Schematic illustration of the regular and catch trials in Experiment 1. A bar was rotated regularly with a step of $18^{\circ}$.

\section{Experiment 2}

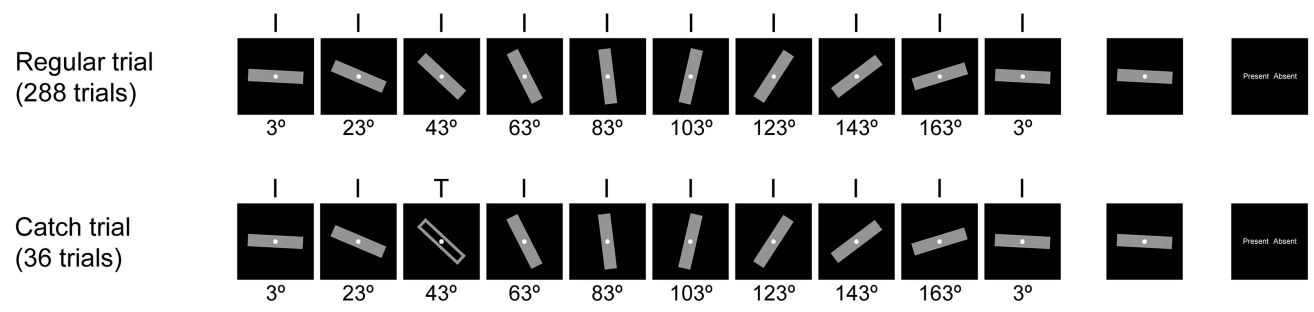

I: Inducing stimulus T: Target stimulus

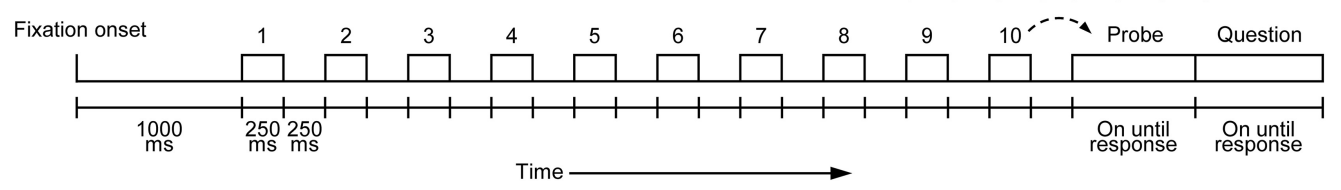

FIGURE 2 | Schematic illustration of the regular and catch trials in Experiment 2. A bar was rotated regularly with a step of $20^{\circ}$.

sources of P2 are still less well understood compared to those of other VEPs, this finding appears to be consistent with a non-human neuroimaging finding suggesting the involvement of lower visual areas (i.e., monkey V2) in the P2 homolog (Metha et al., 2000). The involvement of lower visual areas in P2 is interesting, since the neural sources of temporally earlier VEPs such as P1 at around $110 \mathrm{~ms}$ were localized in higher visual areas including the dorsal and ventral extrastriate cortices (Clark et al., 1995; Di Russo et al., 2002). To explain this paradox, P2 has proposed to be a sign of delayed reactivation of lower visual areas via reentrant feedback projections from higher areas (Di Russo et al., 2003, 2008). Based on these previous findings, prediction suppression of central P2 (Kimura and Takeda, 2015) is best assumed to represent reduced delayed reactivation of lower visual areas around the striate and prestriate cortices. This assumption is consistent with human neuroimaging findings that automatic prediction based on sequential regularities resulted in suppressed activation in lower visual areas including the striate cortex, whereas activation in higher visual areas including the dorsal extrastriate cortex was not affected (Alink et al., 2010) and non-human neuroimaging findings that automatic prediction based on sequential regularities resulted in markedly suppressed activation in lower visual areas (i.e., monkey V2) rather than higher visual areas (Vergnieux and Vogels, 2020; see also Kaposvari et al., 2018).

Taken together, the present study expected that participants who exhibited greater RM may show smaller central P2 in response to inducing stimuli; in other words, the magnitudes of RM and central P2 would show a negative correlation. 


\section{EXPERIMENT 1}

The experiment reported here was conducted with multiple purposes, and included trials that were not related to the present purpose (i.e., irregular trials; see Materials and Methods). Data in the irregular trials have already been reported in another paper (Kimura, 2018). Data reported in this paper have not been reported elsewhere.

\section{Materials and Methods Participants}

Thirty-five healthy adults (32 males, 3 females; mean age 22.5 years; age range $19-32$ years) participated in this experiment. All participants had normal or corrected-to-normal vision. Thirty-three participants were right-handed and two were lefthanded. Written informed consent was obtained from each participant after the nature of the study had been explained. The experiment was approved by the Safety and Ethics committee of the National Institute of Advanced Industrial Science and Technology (AIST).

\section{Stimuli and Procedure}

The experiment was controlled by MATLAB (MathWorks) on Mac OSX with the Psychophysics Toolbox (Brainard, 1997; Pelli, 1997). All visual stimuli were presented on a 17 -inch cathode ray tube display (Sony, Trinitron Multiscan G220) at a viewing distance of about $57 \mathrm{~cm}$.

The experiment consisted of three types of trials (i.e., regular, irregular, and catch trials). Figure 1 shows an illustration of the regular and catch trials; the irregular trials are not related to the present purpose and therefore are not illustrated in Figure 1. The regular trial was included to measure RM and the catch trial was included to ensure that participants kept observing the stimulus sequence. Each trial began with the onset of a gray fixation circle $\left(42.3 \mathrm{~cd} / \mathrm{m}^{2}\right.$; diameter of $\left.0.3^{\circ}\right)$, which was continuously visible on the display. At $1000 \mathrm{~ms}$ after fixation onset, a stimulus sequence consisting of 10 presentations of a bar appeared. In the regular trial, a gray-filled bar $\left(9.2 \mathrm{~cd} / \mathrm{m}^{2}\right.$; width of $0.9^{\circ} \times$ height of $\left.5.7^{\circ}\right)$ was rotated regularly with a step of $18^{\circ}$ (i.e., inducing stimuli). In the catch trial, a gray-filled bar was rotated regularly, but at any of the 10 positions, it was replaced with a gray-unfilled bar (i.e., target stimuli). In all trials, each stimulus was presented for $250 \mathrm{~ms}$ and the inter-stimulus interval, where only the fixation circle was presented, was $250 \mathrm{~ms}$. Note that 10 presentations of inducing stimuli would not necessarily be needed to obtain RM, given that three, four, or at most five presentations of inducing stimuli are common in RM studies. In the present study, 10 presentations were adopted to ensure that prediction had been fully stabilized by the time probe was presented.

This stimulus sequence was followed by a probe. The orientation of the probe was either the same as or slightly different than that of the final (i.e., tenth) inducing stimulus (i.e., $-8^{\circ},-6^{\circ},-4^{\circ},-2^{\circ}, 0^{\circ},+2^{\circ},+4^{\circ},+6^{\circ}$, or $+8^{\circ}$ ). Here, the participants judged whether the orientations of the final inducing stimulus and the probe were the same or different, by pressing either the left or right response button. Mapping of same/different judgments and left/right buttons was fixed throughout the experiment for each participant and counterbalanced across participants. The probe was presented until the participant's response.

The participant's response was immediately followed by a question display consisting of the words "Present" and "Absent." Here, the participants judged whether the target stimulus (i.e., a gray-unfilled bar presented only in the catch trial) was presented or not, by pressing either the left or right response button beside the words on the display. The side on which the words were presented was varied randomly across trials, with a constraint that two possible arrangements (i.e., "Present" on the left and "Absent" on the right, and vice versa) were equally presented within an experiment. The question display was presented until the participant's response, which was immediately followed by a blank screen for $2000 \mathrm{~ms}$.

The experiment included 180 regular trials and 40 catch trials, which were arranged in random order. In the 180 regular trials, 20 trial types, defined by the combination of 10 orientations of the first inducing stimulus (i.e., from $5^{\circ}$ to $167^{\circ}$ with a step of $18^{\circ}$ ) and two directions of regular rotation (i.e., clockwise and counterclockwise), were presented in nine trials each. In these 180 trials, nine angular differences between the final inducing stimulus and the probe (i.e., $-8^{\circ},-6^{\circ},-4^{\circ},-2^{\circ}, 0^{\circ},+2^{\circ}$, $+4^{\circ},+6^{\circ}$, and $+8^{\circ}$ ) were assigned with equal probabilities. Note that the 10 orientations of the first inducing stimulus were used to keep the physical attributes of inducing stimuli presented at each of the 10 positions in a stimulus sequence on average the same. That is, at each of 10 positions, 10 orientations were presented 18 times each.

In the 40 catch trials, the same 20 trial types, defined by the combination of 10 orientations of the first inducing stimulus (i.e., from $5^{\circ}$ to $167^{\circ}$ with a step of $18^{\circ}$ ) and two directions of regular rotation (i.e., clockwise and counterclockwise), were presented in two trials each. In these 40 trials, the target stimulus was presented at each of 10 positions with equal probability. The orientations of the final inducing stimulus and the probe were always the same.

The participants performed the task while seated in a chair in a sound-attenuated, dimly lit room. Before the start of the experiment, the participants were given instructions about the same/different judgment. They were instructed to judge whether the orientations of the tenth stimulus and a subsequent probe were the same or different, as accurately as possible. They were also instructed to count stimuli so that the tenth stimulus could be properly compared with the probe. The speed of their response was not stressed. Here, they were explicitly informed that the angular difference would be $-8^{\circ},-6^{\circ},-4^{\circ},-2^{\circ}, 0^{\circ},+2^{\circ}$, $+4^{\circ},+6^{\circ}$, or $+8^{\circ}$; this was intended to help the participants understand that the angular difference would be quite small. However, they were not informed about the ratio of "same" and "different" trials. Information regarding the nine angular differences might have led participants to expect the probability of each angular difference to be about $11 \%$. However, such expectation is unlikely to significantly affect the magnitude of $\mathrm{RM}$, although it may affect the overall probability of making a "same" response (Hubbard and Lange, 2010). Finally, it was emphasized that they should make a "same" response only 
when they believed that the orientations were exactly the same (Freyd and Finke, 1985).

Next, the participants were given instructions about the present/absent judgment. They were instructed to judge whether or not an unfilled stimulus was presented, as accurately as possible. The speed of their response was not stressed. Here, they were explicitly informed that the unfilled stimulus could appear at any of 10 positions in the stimulus sequence. However, they were not informed about the ratio of "present" and "absent" trials. It was emphasized that they should keep observing the stimulus sequence to perform this task adequately.

Finally, the participants were instructed to minimize any eye movements and blinks when the stimulus sequence was presented. After these instructions, the participants performed 20-40 practice trials, and then started the experiment.

\section{Recordings}

The electroencephalogram (EEG) was recorded with a digital amplifier (Nihon-Kohden, Neurofax EEG1200) and silver-silver chloride electrodes placed at 27 scalp sites (Fp1, Fp2, F7, F3, Fz, F4, F8, FCz, T7, C3, Cz, C4, T8, CPz, P7, P3, Pz, P4, P8, PO7, $\mathrm{PO} 3, \mathrm{POz}, \mathrm{PO} 4, \mathrm{PO} 8, \mathrm{O} 1, \mathrm{Oz}$, and $\mathrm{O} 2$ according to the extended International 10-20 System). All electrodes were referenced to the nose tip. To monitor blinks and eye movements, vertical and horizontal electrooculograms (EOGs) were also recorded with two electrodes above and below the right eye and two electrodes at the right and left outer canthi of the eyes, respectively. The ground electrode was attached to the forehead. The impedance of all electrodes was kept below $5 \mathrm{k} \Omega$. The EEG and EOG signals were bandpass-filtered online at $0.016-300 \mathrm{~Hz}$ and digitized at a sampling rate of $1000 \mathrm{~Hz}$.

The digitized signals were then analyzed by MATLAB (MathWorks) with EEGLAB toolbox (Delorme and Makeig, 2004) and ERPLAB Toolbox (Lopez-Calderon and Luck, 2014). The EEG and EOG signals were bandpass-filtered using a noncausal Butterworth infinite impulse response filter with halfamplitude cutoffs at 0.1 and $30 \mathrm{~Hz}$ and a roll-off of $12 \mathrm{~dB} /$ octave. The EEG and EOG signals time-locked to the onset of inducing stimuli were extracted. The extracted epochs were $600 \mathrm{~ms}$ (i.e., from -100 to $500 \mathrm{~ms}$ relative to the onset of inducing stimuli). An independent component analysis (Delorme and Makeig, 2004) was performed to remove artifacts derived from blinks and eye movements. The epochs were then baseline-corrected relative to the initial $100-\mathrm{ms}$ interval (i.e., from -100 to $0 \mathrm{~ms}$ relative to the onset of inducing stimuli).

For each participant, the EEG signals in the regular trials were averaged for four categories: i.e., inducing stimuli (1) at the first position, (2) at the second, third, and fourth positions, (3) at the fifth, sixth, and seventh positions, and (4) at the eighth, ninth, and tenth positions. VEPs elicited by inducing stimuli at the first position were separately averaged, in consideration of their special morphologies reflecting initial-orienting reaction (Kenemans et al., 1989). VEPs elicited by inducing stimuli at the second-tenth positions were separated for three categories, to explore the time course of the correlation of RM and VEPs, while meeting ideal averaging numbers for VEPs (i.e., about 400 times, Luck, 2005). Note that the physical attributes of the inducing stimuli for these four position categories were on average kept the same. Epochs during which the signal change exceeded \pm 80 $\mu \mathrm{V}$ on any of the EEG or EOG electrodes were excluded from averaging. As a result, the number of epochs averaged for the first, second-fourth, fifth-seventh, and eighth-tenth positions was, on average, $170.8(S D=10.2), 524.5(20.9), 530.1(14.7)$, and 532.8 (10.4), respectively.

\section{Data Analysis \\ Magnitude of RM}

For each participant, the percentages of "same" responses in the regular trials were calculated for nine position categories defined by the angular difference between the final inducing stimulus and the probe and its relation to the direction of regular rotation (i.e., backward $8^{\circ}$, backward $6^{\circ}$, backward $4^{\circ}$, backward $2^{\circ}$, same, forward $2^{\circ}$, forward $4^{\circ}$, forward $6^{\circ}$, and forward $8^{\circ}$ ). Next, for each participant, the magnitude of RM was estimated by a standard formula for calculating the mean position of a probe judged as "same" (Freyd and Jones, 1994; Hayes and Freyd, 2002; Munger and Minchew, 2002). In this calculation, each "same" response was weighted by the position of the probe, and the average of these weighted "same" responses was estimated to be the magnitude of RM. ${ }^{2}$ To confirm the occurrence of RM, the measured values were compared to zero with a one-tailed $t$-test; the statistical threshold was $p<0.05$.

\section{Target detection}

For each participant, the percentage of "present" responses in the catch trials (i.e., hit rate) and those of "absent" responses in the regular trials (i.e., correct rejection rates) were calculated.

\section{Magnitude of VEPs}

For each participant, the magnitudes of VEPs elicited by inducing stimuli in the regular trials were estimated by calculating the mean amplitudes of the occipito-temporal P1, frontal N1, occipito-temporal N1, and central P2 for the four position categories (i.e., first, second-fourth, fifth-seventh, and eighthtenth positions). The time windows of these VEPs for the second-fourth, fifth-seventh, and eighth-tenth positions were determined to be the 40 -ms windows centered on the peaks in the grand-average VEPs in which the three position categories were collapsed; this procedure was chosen to avoid possible biases among the three position categories (Luck, 2014). As a result, the time windows were determined as follows: within the 90-130 ms time window at the PO8 electrode site for occipito-temporal P1, within the 118-158 ms time window at the Fz electrode site for frontal N1, within the 148-188 ms time window at the PO8 electrode site for occipito-temporal N1, and within the 178$218 \mathrm{~ms}$ time window at the $\mathrm{Cz}$ electrode site for central P2

\footnotetext{
${ }^{2}$ In a certain participant, if the mean percentages of "same" response in the backward $8^{\circ}$, backward $6^{\circ}$, backward $4^{\circ}$, backward $2^{\circ}$, same, forward $2^{\circ}$, forward $4^{\circ}$, forward $6^{\circ}$, and forward $8^{\circ}$ conditions were $0,9,12,53,87,90,78,45$, and $12 \%$, respectively, then the sum of the products of the percentage of "same" responses and the distance of the probe from true-same was calculated (i.e., $0 *(-8)+9 *(-6)+12 *(-4)+53^{*}(-2)+87^{*}(0)+90^{*}(+2)+78^{*}(+4)+45^{*}$ $\left.(+6)+12^{*}(+8)=650\right)$, and the obtained value (i.e., 650) was divided by the sum of the percentages of the same responses (i.e., $0+9+12+53+87+90+78+45+12=386)$. This resulted in the magnitude of RM of $1.68^{\circ}$.
} 
(Table 1). The time windows for the first position were separately determined as the 40-ms windows centered on the peaks in the grand-average VEPs for the first position. As a result, the time windows were determined as follows: within the 94-134 ms time window at the PO8 electrode site for occipito-temporal P1, within the 119-159 ms time window at the Fz electrode site for frontal $\mathrm{N} 1$, within the 150-190 ms time window at the PO8 electrode site for occipito-temporal N1, and within the 212-252 ms time window at the $\mathrm{Cz}$ electrode site for central P2 (Table 1).

\section{Correlations between RM and VEPs}

The correlation between the magnitudes of RM and VEPs (i.e., occipito-temporal P1, frontal N1, occipito-temporal N1, and central P2 for the four position categories) were assessed by Spearman's correlation analyses (two-tailed); the statistical threshold was $p<0.05$. Spearman's correlation analysis was chosen here, since the relationship between RM and VEPs was assumed to be not necessarily linear.

\section{Results}

Figure $3 \mathbf{A}$ shows the mean (black line) and individual (gray lines) percentages of "same" responses. Figure $\mathbf{3 B}$ shows the mean (black line) and individual (gray lines) magnitudes of RM. The individual magnitudes of $\mathrm{RM}$ ranged from $0.55^{\circ}$ to $3.02^{\circ}$. The mean magnitude of $\mathrm{RM}$ was $1.86^{\circ}(S D=0.68)$. A one-tailed $t$-test revealed a significant occurrence of $\mathrm{RM}[t(34)=16.31, p<0.001$, $d=2.76]$.

The mean hit rate in the catch trial was 95.1\% ( $S D=8.4)$. The mean correct rejection rate in the regular trial was $98.9 \%$ $(S D=1.1)$.

Figure 4A shows VEPs elicited by the inducing stimuli in the regular trials for the first (red lines), second-fourth (blue lines), fifth-seventh (green lines), and eighth-tenth positions (purple lines). Figure 4B shows topographical maps of VEPs within the time windows listed in Table 1. Typical waveforms consisting of occipito-temporal P1, frontal N1, occipito-temporal N1, and central P2 were observed. Figure 4C shows the mean (black lines) and individual (gray lines) magnitudes of these VEPs, calculated as the mean amplitude according to the time windows and electrodes sites listed in Table $\mathbf{1 .}$

Figure 5 shows the relationship between the magnitudes of RM and VEPs (i.e., occipito-temporal P1, frontal N1, occipitotemporal N1, and central P2 for the four position categories). Spearman's correlation analysis (two-tailed) revealed significant negative correlations between the magnitudes of RM and central

TABLE 1 | Time windows for calculating mean amplitudes of VEPs in Experiment 1.

\begin{tabular}{lcc}
\hline & Position 1 & Positions 2-4, 5-7, and 8-10 \\
\hline Occipito-temporal P1 & $94-134 \mathrm{~ms} \mathrm{(PO8)}$ & $90-130 \mathrm{~ms}$ (PO8) \\
Frontal N1 & $119-159 \mathrm{~ms}(\mathrm{Fz})$ & $118-158 \mathrm{~ms}(\mathrm{Fz})$ \\
Occipito-temporal N1 & $150-190 \mathrm{~ms}(\mathrm{PO} 8)$ & $148-188 \mathrm{~ms}$ (PO8) \\
Central P2 & $212-252 \mathrm{~ms} \mathrm{(Cz)}$ & $178-218 \mathrm{~ms}(\mathrm{Cz})$
\end{tabular}

P2 for the fifth-seventh $(\rho=-0.35 ; p<0.05)$ and eighth-tenth positions $(\rho=-0.39 ; p<0.05) .^{3}$

\section{Discussion}

The results regarding the same/different judgment showed that $\mathrm{RM}$ robustly occurred in the regular trials. This is highly consistent with previous RM findings (Freyd and Finke, 1984, 1985). The results regarding target detection showed that the hit rates in the catch trial as well as the correct rejection rates in the regular trial were high, ensuring that the participants kept observing the stimulus sequence. The results regarding VEPs showed that inducing stimuli elicited occipito-temporal $\mathrm{P} 1$, frontal N1, occipito-temporal N1, and central P2 that were comparable to those obtained with regularly rotated bars (Kimura and Takeda, 2015). For the correlation between RM and VEPs, the magnitude of RM was negatively correlated with the magnitude of central P2; participants who showed a smaller P2 tended to exhibit greater RM. This seems to be consistent with a previous finding that the suppression of central P2 would be a neural effect that would specifically emerge when the current and predicted positions of an object successfully matched (Kimura and Takeda, 2015). In contrast to central P2, the magnitude of RM was not correlated with the magnitude of occipito-temporal P1, frontal $\mathrm{N} 1$, and occipito-temporal N1. Given a previous finding that these VEPs were not sensitive to successful matching between the current and predicted positions of a visual object (Kimura and Takeda, 2015), the null correlation seems to be reasonable.

\section{EXPERIMENT 2}

To test the replicability and robustness of the negative correlation between the magnitudes of RM and central P2, the same analyses were performed on data obtained in another experiment where a bar was regularly rotated with a different angular step (i.e., $20^{\circ}$; cf. $18^{\circ}$ in Experiment 1). Similar to Experiment 1, the experiment reported here was conducted with multiple purposes, and included trials that were not related to the present purpose (i.e., irregular trials; see Materials and Methods). Data in the irregular condition will be reported elsewhere. Data reported in this paper have not been reported elsewhere.

\section{Materials and Methods Participants}

Thirty-seven healthy adults (26 males, 11 females; mean age 23.3 years; age range $20-33$ years) participated in this experiment; three participants had also participated in Experiment 1. All

\footnotetext{
${ }^{3}$ Given that VEPs for the first position showed a large and sustained occipitotemporal positivity at around $200-400 \mathrm{~ms}$ (see Figures $\mathbf{4 A , B}$ ), one may be interested in the relationship between RM and the occipito-temporal positivity. So, an exploratory analysis was made for the correlation between the magnitudes of RM and the occipito-temporal positivity (mean amplitudes were calculated with the time window of $240-280 \mathrm{~ms}$ at PO8 electrode). The results showed that the magnitude of the positivity was not significantly correlated with the magnitude of RM; the first (rho $=0.06, p=0.74$ ), second-forth ( $r h o=0.10, p=0.57$ ), fifth-seventh $(r h o=0.00, p=0.99)$, and eighth-tenth positions $(r h o=-0.06$, $p=0.72$ ).
} 


\section{Experiment 1}

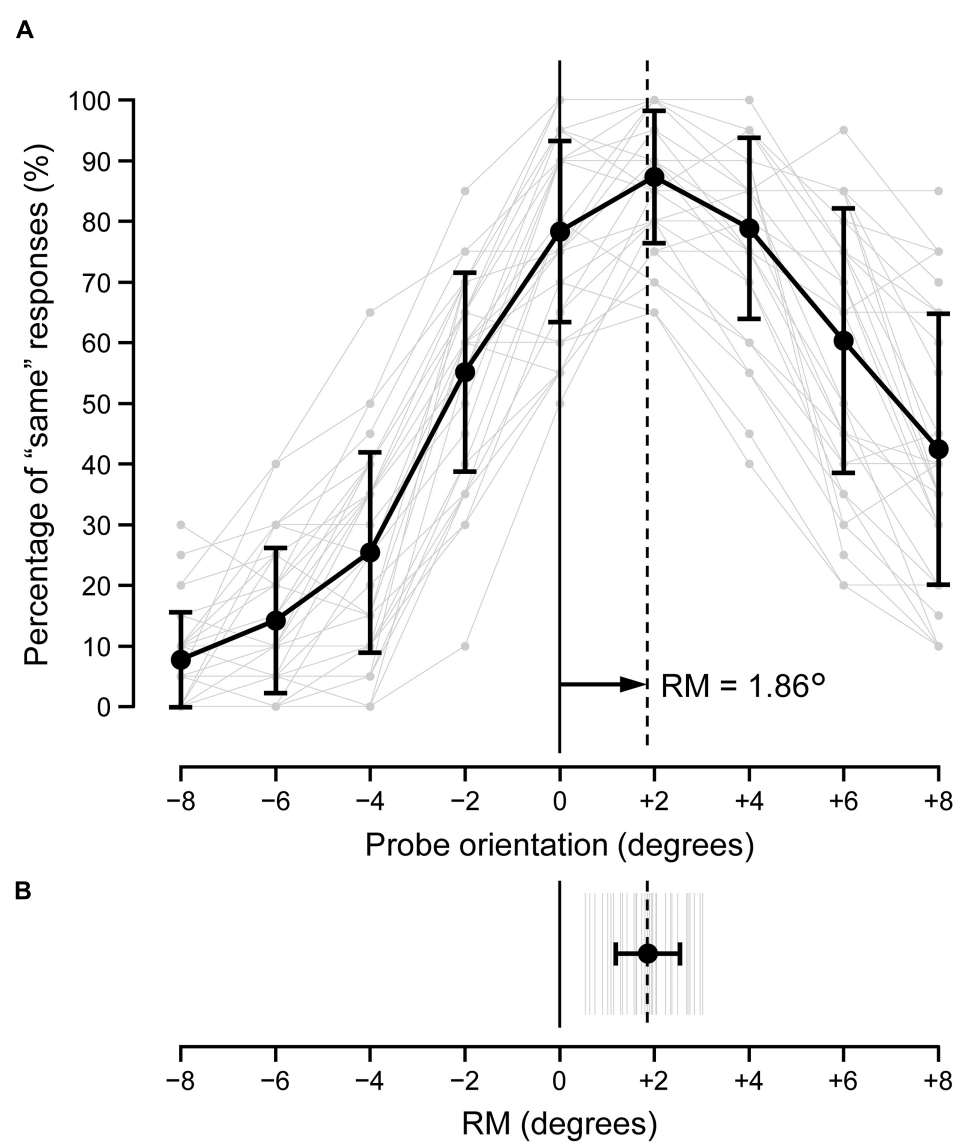

FIGURE 3 | (A) Mean (black) and individual (gray) percentages of "same" responses for the nine probe-orientation categories in the regular trials. Error bars indicate $S D$. (B) Mean (black) and individual (gray) magnitudes of RM. Error bars indicate SD.

participants had normal or corrected-to-normal vision. Thirtysix participants were right-handed and one was left-handed. Written informed consent was obtained from each participant after the nature of the study had been explained. The experiment was approved by the Safety and Ethics committee of the National Institute of Advanced Industrial Science and Technology (AIST).

\section{Stimuli and Procedure}

The stimuli and procedure were the same as those in Experiment 1, except for the following points. The experiment was comprised of three types of trials (i.e., regular, irregular, and catch trials). Figure 2 shows an illustration of the regular and catch trials. In the regular trial, a gray-filled bar was rotated regularly with a step of $20^{\circ}$ (i.e., inducing stimuli). In the catch trial, a gray-filled bar was rotated regularly, but at any of the 10 positions, it was replaced by a gray-unfilled bar (i.e., target stimuli).

The experiment included 288 regular trials and 36 catch trials, which were arranged in random order. The direction of regular rotation was fixed throughout the experiment for each participant and counterbalanced across the participants; for half of the participants (i.e., 18 participants), the direction of regular rotation was clockwise, and for the other half of the participants (i.e., 19 participants), the direction of regular rotation was counterclockwise.

In the 288 regular trials, 36 trial types that were defined by 36 orientations of the first inducing stimulus (i.e., from $3^{\circ}$ to $178^{\circ}$ with a step of $5^{\circ}$ ) were presented in eight trials each. In these 288 trials, nine angular differences between the final inducing stimulus and the probe (i.e., $-8^{\circ},-6^{\circ},-4^{\circ},-2^{\circ}, 0^{\circ},+2^{\circ}$, $+4^{\circ},+6^{\circ}$, and $+8^{\circ}$ ) were assigned with equal probabilities. Note that the 36 orientations of the first stimulus were used to keep the physical attributes of inducing stimuli presented at each of the 10 positions in a stimulus sequence on average the same. Thus, at each of the 10 positions, 36 orientations were presented eight times each.

In the 36 catch trials, the same 36 trial types that were defined by 36 orientations of the first stimulus (i.e., from $3^{\circ}$ to $178^{\circ}$ with a step of $5^{\circ}$ ) were presented in one trial each. In these 36 trials, the target stimulus was presented at each of 10 positions with almost equal probability. 


\section{Experiment 1}

A
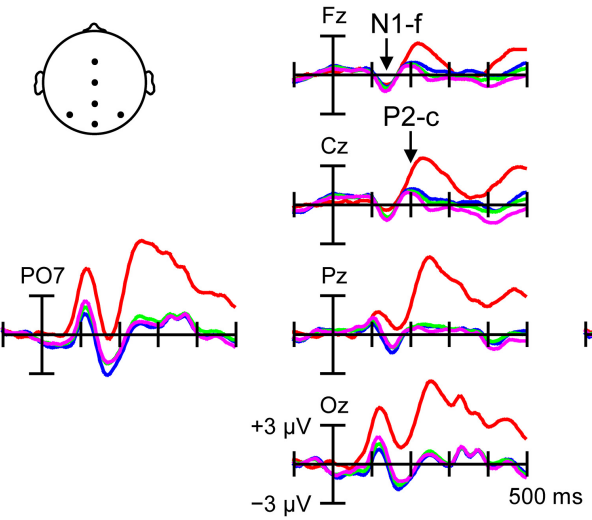

B

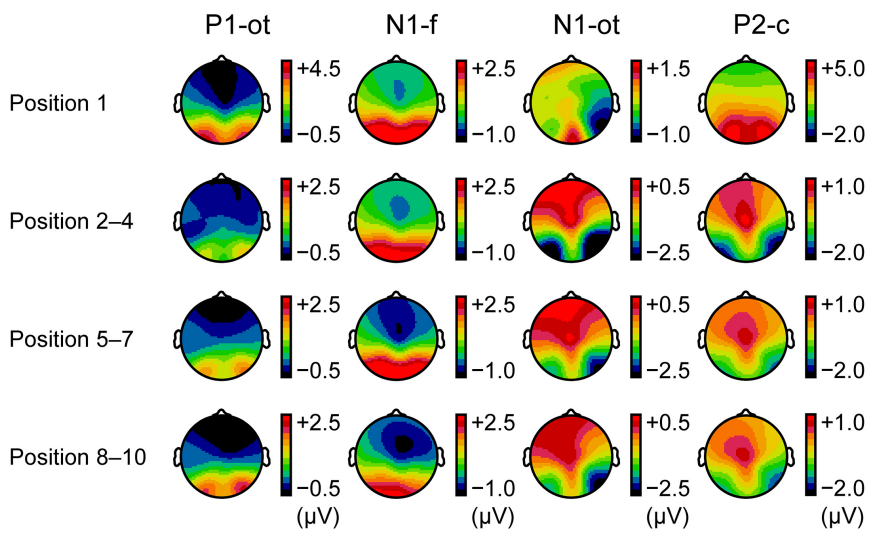

C
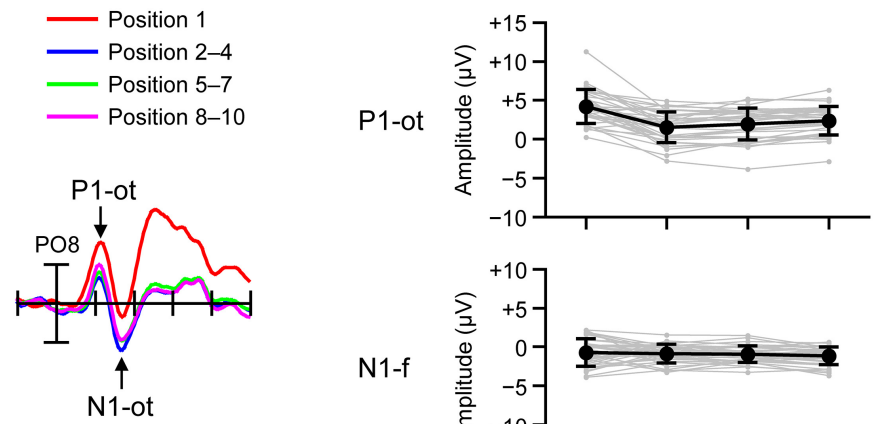

N1-f
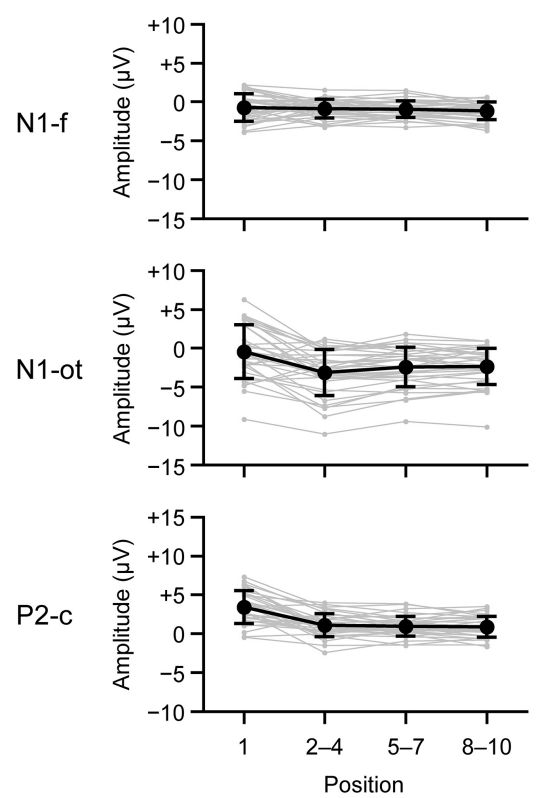

FIGURE 4 | (A) VEPs elicited by inducing stimuli presented at the first position (red lines), second, third, and fourth positions (blue lines), fifth, sixth, and seventh positions (green lines), and eighth, ninth, and tenth positions (purple lines) in the regular trials. (B) Topographical maps of VEPs. (C) Mean (black) and individual (gray) magnitudes of VEPS. P1-ot: occipito-temporal P1, N1-f: frontal N1, N1-ot: occipito-temporal N1, P2-c: central P2. Error bars indicate SD.

\section{Recordings}

The recording parameters were the same as those in Experiment 1. As a result, the number of epochs averaged for the first, second-fourth, fifth-seventh, and eighth-tenth positions was, on average, $280.4(S D=12.1), 851.2(29.8), 856.2(21.1)$, and 857.8 (10.7), respectively.

\section{Data Analysis}

\section{Magnitude of RM}

The data analysis was the same as that in Experiment 1.

\section{Target detection}

The data analysis was the same as that in Experiment 1.

\section{Magnitude of VEPs}

The data analysis was the same as that in Experiment 1, except for the time windows for calculating the mean amplitudes of VEPs. The time windows of VEPs for the second-fourth, fifthseventh, and eighth-tenth positions were determined as follows: within the 89-129 ms time window at the PO8 electrode site for occipito-temporal P1, within the 111-151 ms time window at the $\mathrm{Fz}$ electrode site for frontal $\mathrm{N} 1$, within the 146-186 ms time window at the PO8 electrode site for occipito-temporal N1, and within the 174-214 ms time window at the $\mathrm{Cz}$ electrode site for central P2 (Table 2). The time windows of VEPs for the first position were determined as follows: within the 100-140 ms time window at the PO8 electrode site for occipito-temporal P1, within the 114-154 ms time window at the Fz electrode site for frontal $\mathrm{N} 1$, within the 151-191 ms time window at the PO8 electrode site for occipito-temporal N1, and within the 200-240 ms time window at the $\mathrm{Cz}$ electrode site for central P2 (Table 2).

\section{Correlations between RM and VEPs}

The analysis was the same as that in Experiment 1.

\section{Results}

Figure 6A shows the mean (black line) and individual (gray lines) percentages of "same" responses. Figure 6B shows the mean (black line) and individual (gray lines) magnitudes of RM. The individual magnitudes of $\mathrm{RM}$ ranged from $0.07^{\circ}$ to $3.65^{\circ}$. The mean magnitude of $\mathrm{RM}$ was $2.06^{\circ}(S D=0.80)$. A one-tailed $t$-test revealed a significant occurrence of $\mathrm{RM}[t(36)=15.73, p<0.001$, $d=2.59$ ]. 


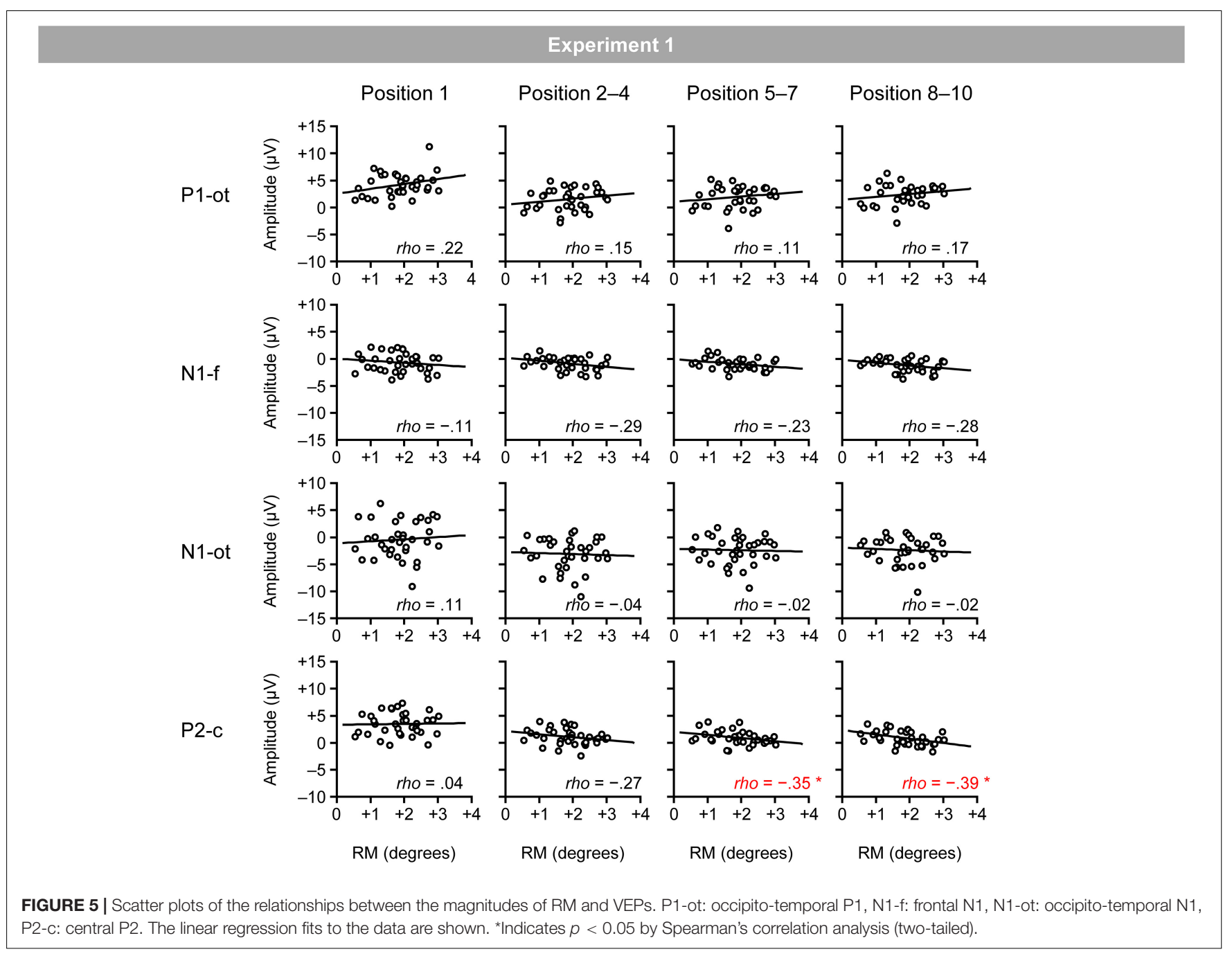

The mean hit rate in the catch trial was 94.7\% ( $S D=6.2)$. The mean correct rejection rate in the regular trial was $98.6 \%$ $(S D=1.1)$.

Figure 7A shows VEPs elicited by the inducing stimuli in the regular trials for the first (red lines), second-fourth (blue lines), fifth-seventh (green lines), and eighth-tenth positions (purple lines). Figure 7B shows topographical maps of VEPs within the time windows listed in Table 2. Figure 7C shows the mean (black lines) and individual (gray lines) magnitudes of VEPs, calculated as the mean amplitude according to the time windows and electrodes sites listed in Table 2.

TABLE 2 | Time windows for calculating mean amplitudes of VEPs in Experiment 2.

\begin{tabular}{lcc}
\hline & Position 1 & Positions 2-4, 5-7, and 8-10 \\
\hline Occipito-temporal P1 & $100-140 \mathrm{~ms} \mathrm{(PO8)}$ & $89-129 \mathrm{~ms}$ (PO8) \\
Frontal N1 & $114-159 \mathrm{~ms} \mathrm{(Fz)}$ & $111-151 \mathrm{~ms}(\mathrm{Fz})$ \\
Occipito-temporal N1 & $151-191 \mathrm{~ms}$ (PO8) & $146-186 \mathrm{~ms}$ (PO8) \\
Central P2 & $200-240 \mathrm{~ms}(\mathrm{Cz})$ & $174-214 \mathrm{~ms}(\mathrm{Cz})$
\end{tabular}

Figure 8 shows the relationship between the magnitudes of RM and VEPs. Spearman's correlation analysis (two-tailed) revealed significant negative correlations between the magnitudes of RM and central P2 for the second-fourth ( $\rho=-0.39 ; p<0.05)$, fifth-seventh $(\rho=-0.40 ; p<0.05)$, and eighth-tenth positions $(\rho=-0.54 ; p<0.01)$, as well as a significant negative correlation between the magnitudes of RM and occipito-temporal P1 for the first position $(\rho=-0.38 ; p<0.05){ }^{4}$

\section{Discussion}

As in Experiment 1, RM robustly occurred in the regular trials, and the inducing stimuli elicited occipito-temporal P1, frontal $\mathrm{N} 1$, occipito-temporal N1, and central P2. The magnitude of RM was again negatively correlated with the magnitude of

\footnotetext{
${ }^{4}$ As in Experiment 1, an exploratory analysis for the correlation between the magnitudes of RM and the occipito-temporal positivity (mean amplitudes were calculated with the time window of $240-280 \mathrm{~ms}$ at PO8 electrode) showed that the magnitude of the positivity was not significantly correlated with the magnitude of RM; the first (rho $=0.05, p=0.76$ ), second-forth ( $r h o=0.13, p=0.46$ ), fifth-seventh ( $r h o=0.16, p=0.34$ ), and eighth-tenth positions ( $r h o=0.03$, $p=0.87)$.
} 


\section{Experiment 2}

A

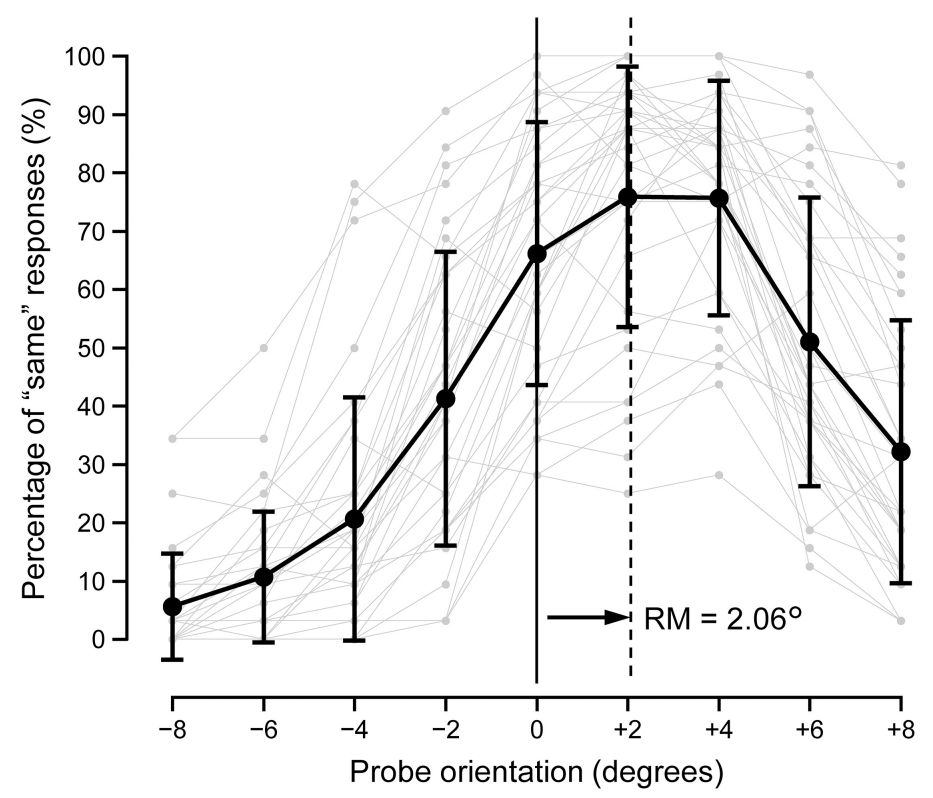

B

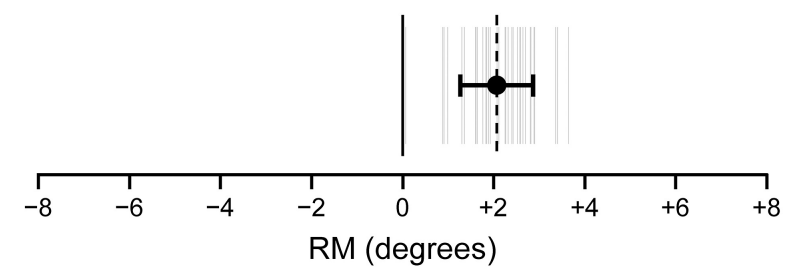

FIGURE 6 | (A) Mean (black) and individual (gray) percentages of "same" responses for the nine probe-orientation categories in the regular trials. Error bars indicate $S D$. (B) Mean (black) and individual (gray) magnitudes of RM. Error bars indicate $S D$.

central P2; participants who showed a smaller P2 tended to exhibit greater RM. Thus, the negative correlation between the magnitudes of RM and central P2 observed in Experiment 1 was clearly replicated in Experiment 2, ensuring the replicability and robustness of the negative correlation between RM and central $\mathrm{P} 2$. In addition to central P2, the magnitude of occipito-temporal P1 for the first position was negatively correlated with the magnitude of RM. However, given that such negative correlation was not observed in Experiment 1 (rather, a tendency of an opposite, positive correlation was observed in Experiment 1), no conclusion could be drawn about this effect.

\section{GENERAL DISCUSSION}

In Experiments 1 and 2, the results regarding the same/different judgment showed that RM clearly occurred in regular trials. This is highly consistent with the previous findings with the conventional RM paradigm with regular rotations of a bar (Freyd and Finke, 1984, 1985) as well as with other types of changes (Kelly and Freyd, 1987; Hayes and Freyd, 2002). The magnitude of RM in Experiment 2 (mean of $2.06^{\circ}$ ) was numerically greater than that in Experiment 1 (mean of $1.86^{\circ}$ ). This could be mainly attributed to the step size of a regular rotations of a bar (i.e., $18^{\circ}$ in Experiment 1 and $20^{\circ}$ in Experiment 2), since the magnitude of RM is proportional to the implied velocity of regular rotations of a bar (Freyd and Finke, 1985; Finke et al., 1986).

In Experiments 1 and 2, the magnitude of RM was negatively correlated with the magnitude of central P2 at around $200 \mathrm{~ms}$ after bar onset; that is, participants who showed a smaller P2 tended to exhibit greater RM. This is consistent with the expectation based on a previous finding that the suppression of central P2 is a neural effect that specifically emerges when the current position of a visual object successfully matches the predicted position of the object based on sequential regularities (Kimura and Takeda, 2015). The negative correlations between the magnitudes of RM and central P2 showed a similar time course in Experiments 1 and 2. That is, the magnitudes of RM and central P2 were not initially correlated at the first position, and they started to be negatively correlated at later positions. These results support the idea that the correlation would be associated with the individual's ability to automatically form a prediction based on sequential regularities and contradict the idea that the correlation between the magnitudes of RM and P2 merely 


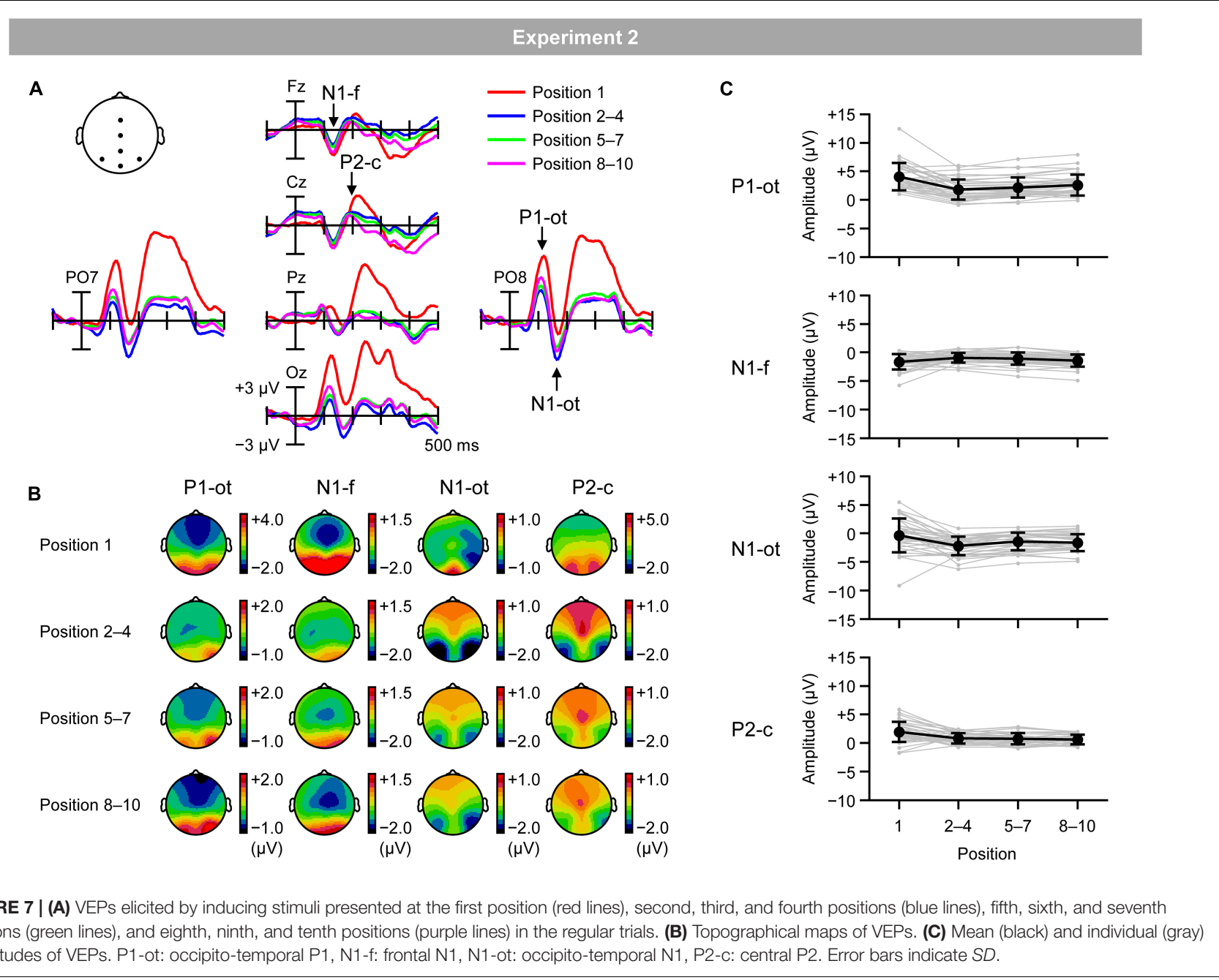

reflects the individual's inherent strength of neural activations represented by $\mathrm{P} 2$.

Although the negative correlations between the magnitudes of RM and central P2 were highly similar between Experiments 1 and 2, there were slight differences between Experiments 1 and 2. The negative correlation appeared earlier in Experiment 2 (i.e., the second-fourth positions) than in Experiment 1 (i.e., the fifth-seventh positions). Also, the negative correlation between the magnitudes of RM and central P2 was more robust (at least in terms of the correlation coefficient) in Experiment 2 than in Experiment 1 . These differences would be mainly attributed to two differences in the experimental design. First, they may be attributed to the greater step size of the regular rotation of a bar in Experiment 2 (i.e., $20^{\circ}$ ) than in Experiment 1 (i.e., $18^{\circ}$ ). Second, they may be attributed to the arrangement of directions of regular rotation. In Experiment 1, directions of regular rotation (i.e., clockwise and counterclockwise) were changed trial-by-trial in a random manner; therefore, only after the second inducing stimulus was presented, the participants could recognize whether the current regular rotation was clockwise or counterclockwise and could predict the orientation of the upcoming inducing stimuli. In contrast, in Experiment 2, the direction of regular rotation (i.e., clockwise or counterclockwise) was fixed for each participant throughout the experiment; therefore, immediately after the first inducing stimulus was presented, the participants could predict the orientation of the upcoming inducing stimuli.

It appears difficult to attribute the negative correlation between RM and central P2 to factors other than prediction. For example, one might consider that the negative correlation may be involved in visual attention to inducing stimuli. However, if the negative correlation was involved in the degree of visual attention, then significant correlations should have also been observed between RM and occipito-temporal P1/N1, since visual attention predominantly affects occipito-temporal P1/N1 (Hillyard and Anllo-Vento, 1998; Luck et al., 2000). The present results of the almost null correlation between RM and P1/N1 are incongruent with this expectation. One might also consider that the negative correlation may be associated with some strategic processes. It has been shown that RM is primarily determined by automatic predictive processes. However, due to the essential requirements of the task (i.e., the same/different judgment), RM may not be free from the effects of strategic 


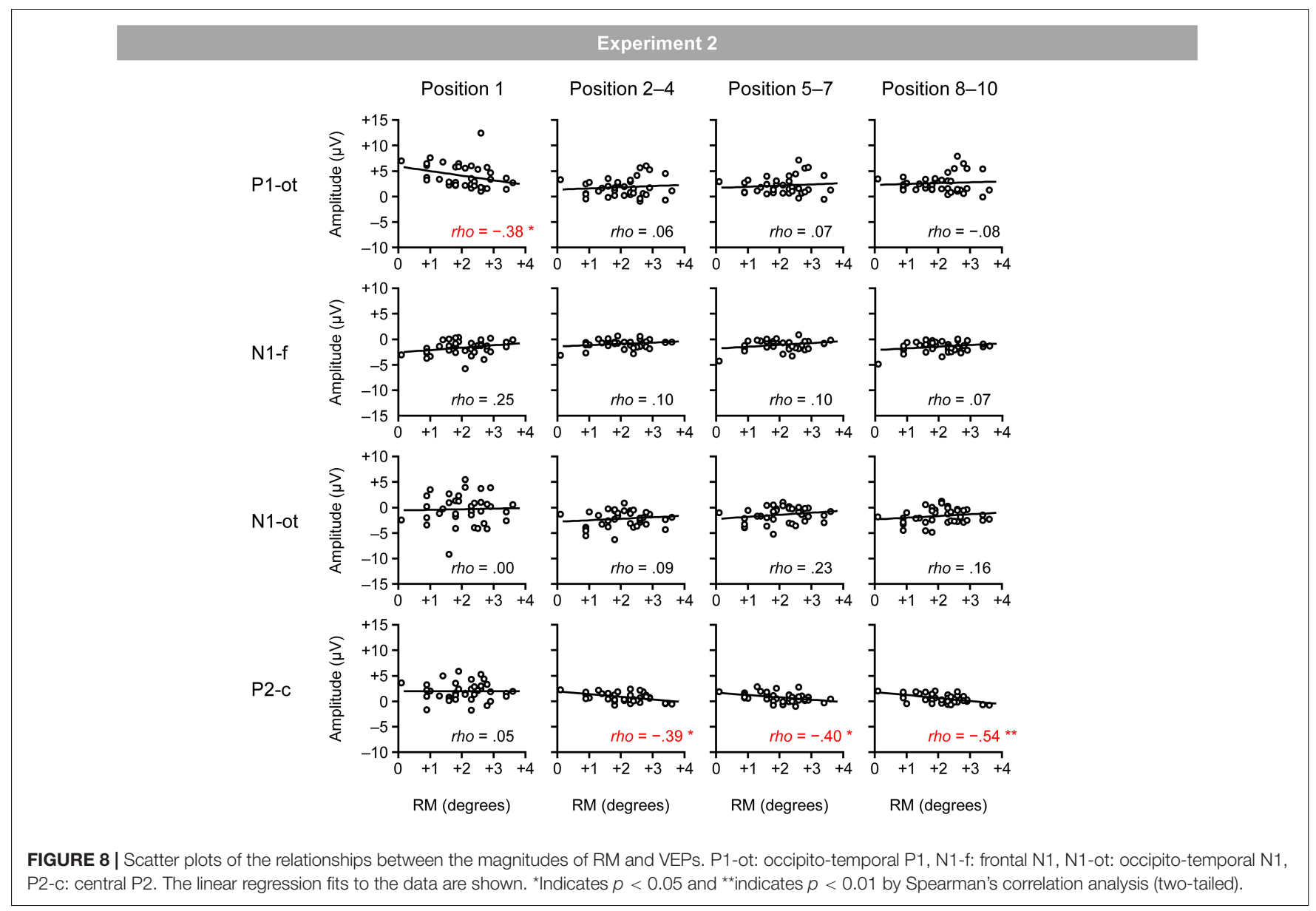

processes such as "cognitive resistance" (i.e., to intentionally stop the forward displacement of a sensory representation to improve the same/different judgment; Finke et al., 1986) and "opposite-acting compensation" (i.e., to strategically change the judgment to compensate for a likely perceptual bias; Joordens et al., 2004). Although such effects of strategic processes could not be completely ruled out, given that the present negative correlation was not limited to the eighth-tenth positions where such strategic processes are expected to be operated, it seems unlikely that the presented negative correlation was related to such strategic processes.

Taken together, the present results suggest that the greater sensory suppression as indicated by smaller central P2 underlies stronger predictive modulation of visual perception as indicated by greater RM. Given the previous findings that neural sources of central P2 were localized around lower visual areas around the striate and prestriate cortices (Capilla et al., 2016; see also Metha et al., 2000) and P2 may be a sign of delayed reactivation of lower visual areas via reentrant feedback projections from higher areas (Di Russo et al., 2003, 2008; see also Olson et al., 2001; Noesselt et al., 2002), the present results would support the notion that the strength of prediction suppression of delayed reactivation of lower visual areas determines the strength of predictive modulation of visual perception. This notion is consistent with that in a human neuroimaging study which demonstrated that successful matching between current visual inputs and predicted visual inputs based on sequential regularities drives less neural activation in the striate cortex, probably via feedback projections from higher visual areas (Alink et al., 2010). From a broader perspective, the present findings appear to be in line with previous findings that the strength of delayed reactivation of lower visual areas such as striate cortex via reentrant feedback projections critically determines perceptual experience and awareness (Lamme et al., 1998; Lamme and Roelfsema, 2000; Tong, 2003; Pak et al., 2020) as well as the hierarchical predictive coding framework which proposes that prior expectations about an upcoming stimulus act as top-down signals that predict the bottom-up input (Rao and Ballard, 1999; Friston, 2005; Summerfield and de Lange, 2014).

The present findings should be treated with caution in three respects. First, the present study used a simple stimulus (i.e., a bar) that changed along with a simple regularity (i.e., rotation). It seems possible that, when observing a more complex stimulus (e.g., face) that changes along with a more complex regularity (e.g., changes in facial features), the main loci of prediction suppression might change (e.g., from lower visual areas to higher areas such as face-responsible inferior temporal cortex, 
Haxby et al., 2000), and the prediction suppression in such higher areas may mainly determine the predictive modulation of visual perception. Second, the present study applied a conventional RM paradigm (Freyd and Finke, 1984, 1985), but there are several different RM paradigms such as those with a still photograph of an object in motion (Freyd, 1983) or a smooth animated motion of an object (Hubbard and Bharucha, 1988). To capture the overall picture of the relationship between prediction suppression based on sequential regularities and predictive modulation of visual perception, the accumulation of studies with a variety of paradigm should be required. Third, the present study did not directly examine the neural sources of central P2. The precise source localization was not a realistic option in the present study, since central P2 was expected to be overlapped by temporally and/or spatially adjacent VEPs. Furthermore, although a previous study reported that the neural sources of central P2 were localized in lower visual areas (Capilla et al., 2016), stimuli used in the previous study (i.e., reversal of a checkerboard pattern) were different from those used in the present study (i.e., discrete presentation of a bar). In future studies, the direct examination of the neural sources should be made with an optimal experimental design by which the predictive suppression of central P2 can be isolated from other neural activities (Kimura and Takeda, 2015).

Finally, this present finding may drive the fundamental question of what factors determine the individual's ability to automatically form a prediction based on sequential regularities. For example, previous RM studies showed that the magnitude of RM can be modulated by domain-specific expertise (e.g., greater $\mathrm{RM}$ for road scenes in experienced compared to inexperienced automobile drivers), suggesting that prediction ability can be improved with expertise (Blättler et al., 2010, 2011). As another approach, a recent study sought clinical factors that determine the magnitude of RM in terms of autistic and schizotypal traits, although a strong factor could not be determined (Tulver et al., 2019). The quest for critical factors that determine an individual's

\section{REFERENCES}

Alink, A., Schwiedrzik, C. M., Kohler, A., Singer, W., and Muckli, L. (2010). Stimulus predictability reduces responses in primary visual cortex. J. Neurosci. 30, 2960-2966. doi: 10.1523/jneurosci.3730-10.2010

Blättler, C., Ferrari, V., Didierjean, A., and Marméche, E. (2011). Representational momentum in aviation. J. Exp. Psychol. Hum. Percept. Perform. 37, 1569-1577. doi: 10.1037/a0023512

Blättler, C., Ferrari, V., Didierjean, A., van Elslande, P., and Marméche, E. (2010). Can expertise modulate representational momentum? Vis. Cogn. 18, 12531273. doi: $10.1080 / 13506281003737119$

Brainard, D. H. (1997). The psychophysics toolbox. Spat. Vis. 10, 433-436. doi: $10.1163 / 156856897 \times 00357$

Capilla, A., Melcón, M., Kessel, D., Calderón, R., Pazo-Álvarez, P., and Carretié, L. (2016). Retinotopic mapping of visual event-related potentials. Biol. Psychol. 118, 114-125. doi: 10.1016/j.biopsycho.2016.05.009

Clark, V. P., Fan, S., and Hillyard, S. A. (1995). Identification of early visual evoked potential generators by retinotopic and topographic analyses. Hum. Brain Mapp. 2, 170-187. doi: 10.1002/hbm.460020306

Delorme, A., and Makeig, S. (2004). EEGLAB: an open source toolbox for analysis of single-trial EEG dynamics including independent component analysis. J. Neurosci. Methods 134, 9-21. doi: 10.1016/j.jneumeth.2003.10.009 prediction abilities would be important for better understanding the mechanisms of visual perception and for establishing possible training/intervention methods to improve prediction abilities.

\section{CONCLUSION}

By measuring VEPs with a conventional RM paradigm, the present study demonstrated the relationship between the strength of predictive modulation of visual perception (as measured by the magnitude of RM) and the strength of prediction suppression of sensory response (as measured by the magnitude of central P2, which is best assumed to represent delayed reactivation of lower visual areas around striate and prestriate cortices via reentrant feedback projections from higher areas).

\section{DATA AVAILABILITY STATEMENT}

The raw data supporting the conclusion of this article will be made available by the authors, without undue reservation.

\section{ETHICS STATEMENT}

The studies involving human participants were reviewed and approved by the Safety and Ethics Committee of the National Institute of Advanced Industrial Science and Technology (AIST). The patients/participants provided their written informed consent to participate in this study.

\section{AUTHOR CONTRIBUTIONS}

The author confirms being the sole contributor of this work and has approved it for publication.

Di Russo, F., Aprile, T., Spitoni, G., and Spinelli, D. (2008). Impaired visual processing of contralesional stimuli in neglect patients: a visual-evoked potential study. Brain 131, 842-854. doi: 10.1093/brain/awm281

Di Russo, F., Martínez, A., and Hillyard, S. A. (2003). Source analysis of eventrelated cortical activity during visuo-spatial attention. Cereb. Cortex 13, 486499. doi: 10.1093/cercor/13.5.486

Di Russo, F., Martínez, A., Sereno, M. I., Pitzalis, S., and Hillyard, S. A. (2002). Cortical sources of the early components of the visual evoked potential. Hum. Brain Mapp. 15, 95-111. doi: 10.1002/hbm.10010

Finke, R. A., and Freyd, J. J. (1985). Transformations of visual memory induced by implied motions of pattern elements. J. Exp. Psychol. Learn. Mem. Cogn. 11, 780-794. doi: 10.1037/0278-7393.11.1-4.780

Finke, R. A., Freyd, J. J., and Shyi, G. C. W. (1986). Implied velocity and acceleration induced transformations of visual memory. J. Exp. Psychol. Gen. 115, 175-188. doi: 10.1037/0096-3445.115.2.175

Freyd, J. J. (1983). The mental representation of movement when static stimuli are viewed. Percept. Psychophys. 33, 575-581. doi: 10.3758/bf03202 940

Freyd, J. J. (1992). “Dynamic representations guiding adaptive behavior," in Time, Action and Cognition: Towards Bridging the Gap, eds F. Macar, V. Pouthas, and W. J. Friedman (Dordrecht: Kluwer Academic Publishers), 309-323. doi: 10.1007/978-94-017-3536-0_32 
Freyd, J. J., and Finke, R. A. (1984). Representational momentum. J. Exp. Psychol. Learn. Mem. Cogn. 10, 126-132.

Freyd, J. J., and Finke, R. A. (1985). A velocity effect for representational momentum. Bull. Psychon. Soc. 23, 443-446. doi: 10.3758/bf03329847

Freyd, J. J., and Jones, K. T. (1994). Representational momentum for a spiral path. J. Exp. Psychol. Learn. Mem. Cogn. 20, 968-976. doi: 10.1037/0278-7393.20.4. 968

Freyd, J. J., Kelly, M. H., and DeKay, M. L. (1990). Representational momentum in memory for pitch. J. Exp. Psychol. Learn. Mem. Cogn. 16, 1107-1117. doi: 10.1037/0278-7393.16.6.1107

Friston, K. J. (2005). A theory of cortical responses. Philos. Trans. R. Soc. Lond. Ser. B Biol. Sci. 360, 815-836.

Haxby, J. V., Hoffman, E. A., and Gobbini, M. I. (2000). The distributed human neural system for face perception. Trends Cogn. Sci. 4, 223-233. doi: 10.1016/ s1364-6613(00)01482-0

Hayes, A. E., and Freyd, J. J. (2002). Representational momentum when attention is divided. Vis. Cogn. 9, 8-27. doi: 10.1080/13506280143000296

Hillyard, S. A., and Anllo-Vento, L. (1998). Event-related brain potentials in the study of visual selective attention. Proc. Natl. Acad. Sci. U.S.A. 95, 781-787.

Hubbard, T. L. (1995). Environmental invariants in the representation of motion: implied dynamics and representational momentum, gravity, friction, and centripetal force. Psychon. Bull. Rev. 2, 322-338. doi: 10.3758/bf03210 971

Hubbard, T. L. (2005). Representational momentum and related displacements in spatial memory: a review of the findings. Psychon. Bull. Rev. 12, 822-851. doi: $10.3758 / \mathrm{bf} 03196775$

Hubbard, T. L., and Bharucha, J. J. (1988). Judged displacement in apparent vertical and horizontal motion. Percept. Psychophys. 44, 211-221. doi: 10.3758/ bf03206290

Hubbard, T. L., and Lange, M. (2010). Prior probabilities and representational momentum. Vis. Cogn. 18, 1063-1087. doi: 10.1080/13506281003665708

Joordens, S., Spalek, T. M., Razmy, S., and van Duijn, M. (2004). A clockwork orange: compensation opposing momentum in memory for location. Mem. Cogn. 32, 39-50. doi: 10.3758/bf03195819

Kaposvari, P., Kumar, S., and Vogels, R. (2018). Statistical learning signals in macaque inferior temporal cortex. Cereb. Cortex 28, 250-266. doi: 10.1093/ cercor/bhw374

Kelly, M. H., and Freyd, J. J. (1987). Explorations of representational momentum. Cogn. Psychol. 19, 369-401. doi: 10.1016/0010-0285(87)90009-0

Kenemans, J. L., Verbaten, M. N., Roelofs, J. W., and Slangen, J. L. (1989). "Initial" and "change-orienting reactions": an analysis based on visual single-trial event-related potentials. Biol. Psychol. 28, 199-226. doi: 10.1016/0301-0511(89) 90001-x

Kimura, M. (2018). Visual mismatch negativity and representational momentum: their possible involvement in the same automatic prediction. Biol. Psychol. 139, 178-185. doi: 10.1016/j.biopsycho.2018.10.015

Kimura, M., and Takeda, Y. (2015). Automatic prediction regarding the next state of a visual object: electrophysiological indicators of prediction match and mismatch. Brain Res. 1626, 31-44. doi: 10.1016/j.brainres.2015.01.013

Lamme, V. A., and Roelfsema, P. R. (2000). The distinct modes of vision offered by feedforward and recurrent processing. Trends Neurosci. 23, 571-579. doi: 10.1016/s0166-2236(00)01657-x

Lamme, V. A., Supèr, H., and Spekreijse, H. (1998). Feedforward, horizontal, and feedback processing in the visual cortex. Curr. Opin. Neurobiol. 8, 529-535. doi: 10.1016/s0959-4388(98)80042-1

Lopez-Calderon, J., and Luck, S. J. (2014). ERPLAB: an open-source toolbox for the event-related potentials. Front. Hum. Neurosci. 8:213. doi: 10.3389/fnhum. 2014.00213

Luck, S. J. (2005). An Introduction to the Event-Related Potential Technique. Cambridge, MA: MIT Press.

Luck, S. J. (2014). An Introduction to the Event-Related Potential Technique, 2nd Edn. Cambridge, MA: MIT Press.
Luck, S. J., Woodman, G. F., and Vogel, E. K. (2000). Event-related potential studies of attention. Trends Cogn. Sci. 4, 432-440.

Mackay, D. M. (1958). Perceptual stability of a stroboscopically lit visual field containing self-luminous objects. Nature 181, 507-508. doi: 10.1038/181507a0

Metha, A. D., Ulbert, I., and Schroeder, C. E. (2000). Intermodal selective attention in monkeys. II: physiological mechanisms of modulation. Cereb. Cortex 10, 359-370. doi: $10.1093 /$ cercor/10.4.359

Munger, M. P., and Minchew, J. H. (2002). Parallels between remembering and predicting an object's location. Vis. Cogn. 9, 177-194. doi: 10.1080/ 13506280143000386

Nijhawan, R. (1994). Motion extrapolation in catching. Nature 370, 256-257. doi: $10.1038 / 370256 \mathrm{~b} 0$

Noesselt, T., Hillyard, S. A., Woldorff, M. G., Schoenfeld, A., Hagner, T., Jäncke, L., et al. (2002). Delay striate cortical activation during spatial attention. Neuron 35, 575-587. doi: 10.1016/s0896-6273(02)00781-x

Olson, I. R., Chun, M. M., and Allison, T. (2001). Contextual guidance of attention: Human intracranial event-related potential evidence for feedback modulation in anatomically early, temporally late stages of visual processing. Brain 124, 1417-1425. doi: 10.1093/brain/124.7.1417

Pak, A., Ryu, E., Li, C., and Chubykin, A. A. (2020). Top-down feedback controls the cortical representation of illusory contours in mouse primary visual cortex. J. Neurosci. 40, 648-660. doi: 10.1523/jneurosci.1998-19.2019

Pelli, D. G. (1997). The VideoToolbox software for visual psychophysics: transforming numbers into movies. Spat. Vis. 10, 437-442. doi: 10.1163/ $156856897 \times 00366$

Rao, R. P. N., and Ballard, D. H. (1999). Predictive coding in the visual cortex: a functional interpretation of some extra-classical receptive-field effects. Nat. Neurosci. 2, 79-87. doi: 10.1038/4580

Summerfield, C., and de Lange, F. P. (2014). Expectation in perceptual decision making: neural and computational mechanisms. Nat. Rev. Neurosci. 15, 745756. doi: $10.1038 / \mathrm{nrn} 3838$

Todorovic, A., and de Lange, F. P. (2012). Repetition suppression and expectation suppression are dissociable in time in early auditory evoked fields. J. Neurosci. 32, 13389-13395. doi: 10.1523/jneurosci.2227-12.2012

Tong, F. (2003). Primary visual cortex and visual awareness. Nat. Rev. Neurosci. 4 , 219-229. doi: 10.1038/nrn1055

Tulver, K., Aru, J., Rutiku, R., and Bachmann, T. (2019). Individual differences in the effects of priors on perception: a multi-paradigm approach. Cognition 187, 167-177. doi: 10.1016/j.cognition.2019.03.008

Verfaillie, K., and d'Ydewalle, G. (1991). Representational momentum and event course anticipation in the perception of implied periodical motions. J. Exp. Psychol. Learn. Mem. Cogn. 17, 302-313. doi: 10.1037/0278-7393. 17.2.302

Vergnieux, V., and Vogels, R. (2020). Statistical learning signals for complex visual images in macaque early visual cortex. Front. Hum. Neurosci. 14:789. doi: $10.3389 /$ fnins.2020.00789

Conflict of Interest: The author declares that the research was conducted in the absence of any commercial or financial relationships that could be construed as a potential conflict of interest.

Publisher's Note: All claims expressed in this article are solely those of the authors and do not necessarily represent those of their affiliated organizations, or those of the publisher, the editors and the reviewers. Any product that may be evaluated in this article, or claim that may be made by its manufacturer, is not guaranteed or endorsed by the publisher.

Copyright $\odot 2021$ Kimura. This is an open-access article distributed under the terms of the Creative Commons Attribution License (CC BY). The use, distribution or reproduction in other forums is permitted, provided the original author(s) and the copyright owner(s) are credited and that the original publication in this journal is cited, in accordance with accepted academic practice. No use, distribution or reproduction is permitted which does not comply with these terms. 